\title{
Quantile Regression Models and Their Applications: A Review
}

\author{
Qi Huang1, Hanze Zhang', Jiaqing Chen ${ }^{3 *}$ and Mengying $\mathrm{He}^{4}$ \\ ${ }^{1}$ School of Law, Wuhan University, Wuhan, Hubei, 430072, PR China \\ ${ }^{2}$ Department of Epidemiology and Biostatistics, College of Public Health, University of South Florida, Tampa, FL 33612, USA \\ ${ }^{3}$ College of Science, Wuhan University of Technology, Wuhan, Hubei, 430070, PR China \\ ${ }^{4}$ Department of Management, College of Business, Texas A\&M University San Antonio, San Antonio, TX 78224, USA
}

\begin{abstract}
Quantile regression (QR) has received increasing attention in recent years and applied to wide areas such as investment, finance, economics, medicine and engineering. Compared with conventional mean regression, QR can characterize the entire conditional distribution of the outcome variable, may be more robust to outliers and misspecification of error distribution, and provides more comprehensive statistical modeling than traditional mean regression. QR models could not only be used to detect heterogeneous effects of covariates at different quantiles of the outcome, but also offer more robust and complete estimates compared to the mean regression, when the normality assumption violated or outliers and long tails exist. These advantages make QR attractive and are extended to apply for different types of data, including independent data, time-to-event data and longitudinal data. Consequently, we present a brief review of QR and its related models and methods for different types of data in various application areas.
\end{abstract}

Keywords: Quantile regression; Check function; Asymmetric laplace distribution; Time-to-event; Longitudinal data

\section{Introduction}

In statistical modeling, regression has been developed to quantify the relationship between dependent variable (outcome) and independent variables (covariates) for over 200 years. The classic regression has been one of the most widely used statistical methods to capture the effects at the mean. These conventional regressions assume that the regression coefficients/covariates effects are constant across the population. However, such average effects are not always of interest in many areas, and sometimes quite heterogeneous. For example, Quantile regression $(\mathrm{QR})$ with applications by exploring the relation of the foreign direct investment and economic growth $[1,2]$ and in "precision health/medicine" $[3,4]$ have been widely adopted in related fields currently. A lot of researchers, economists, financial investors, clinicians and policymakers have showed increasing attention on group differences across the entire population rather than that solely on the average. Mean regression cannot satisfy with all of these needs or requirements.

Developed by Koenker and Bassett in 1978 [5], QR complements and improves the traditional mean regression models. In this situation of homogeneity assumption violated, QR quantifies the heterogeneous effects of covariates through conditional quantiles of the outcome variable, and provides a comprehensive scan of the whole distribution of the outcome. Additionally, it is well known that when asymmetries and heavy tails exist, the sample median (the $50^{\text {th }}$ percentile), one of the best-known example of quantiles, provides a better summary of centrality than the mean. As a consequence, compared to the standard mean regression models, QR is more robust to outliers and more flexible, because the distribution of the outcome does not need to be strictly specified as certain parametric assumptions. Although mean regression-based methods still dominate the statistical modeling field, QR can be viewed as a critical extension and complement when assumptions are violated. Thus, QR has become a subject of intense investigation and application in the past decades.

QR has attracted considerable research interest in decades, and has been widely applied to independent data and time-to-event data. Recently, the use of $\mathrm{QR}$ for longitudinal data has also received increasing attention. This review article is organized to provide a brief overview of QR models and associated statistical methods for these three types of data with applications in different areas.

\section{QR Models for Independent Data}

In analogy with traditional linear regression, QR model for independent data was formally formulated by Koenker and Bassett [5] in 1978 as an extension from the notion of ordinary percentiles. The different QR approaches can be roughly classified into two groups: (i) minimization of weighted absolute deviations, which is a typical inferential method used in QR; and (ii) the maximization of a Laplace likelihood.

The former is based on Koenker and Bassetts work [5], which estimated the conditional median and a full range of other quantile functions by minimizing asymmetrically weighted absolute residuals. Generally, let $y_{i}$ and $x_{i}$ denote the outcome of interest and the corresponding covariate vector for subject $i(i=1, \ldots, n)$, where $y_{i}$ is independent scalar observations of a continuous random variable with common cumulative distribution function (cdf) $F_{y}(\cdot)$. The QR model with $\tau$ th quantile for the response $y_{i}$ given $x_{i}$ takes the form of

$$
Q_{y_{i}}\left(\tau \mid x_{i}\right)=g\left(x_{i}, \beta\right),
$$

where $Q_{y_{i}}(\cdot)=F_{y_{i}}^{-1}(\cdot)$ is the inverse of cdf of $y_{i}$ given $x_{i}$ evaluated at $\tau$ with $0<\tau<1, g(\cdot)$ is a known function. The regression coefficient vector $\beta$ is estimated by minimizing

$$
\sum_{i=1}^{n} \rho_{\tau}\left(y_{i}-g\left(x_{i}, \beta\right)\right),
$$

*Corresponding author: Chen J, College of Science, Wuhan University of Technology, Wuhan, Hubei, 430070, PR China, Tel: 86-27-87163146; E-mail: hyx123@hotmail.com

Received May 30, 2017; Accepted June 16, 2017; Published June 20, 2017

Citation: Huang Q, Zhang H, Chen J, He M (2017) Quantile Regression Models and Their Applications: A Review. J Biom Biostat 8: 354. doi: 10.4172/21556180.1000354

Copyright: ( 2017 Huang Q, et al. This is an open-access article distributed under the terms of the Creative Commons Attribution License, which permits unrestricted use, distribution, and reproduction in any medium, provided the original author and source are credited. 
Where $\rho_{\tau}(\cdot)$ is the check function defined by $\rho_{\tau}(u)=u(\tau-I(u<0))$ and $\mathrm{I}(\cdot)$ denotes the indictor function. A full discussion of this class of methods could be found from many related publications [5-8]. Traditional QR makes minimal assumptions on the form of the error term, which is flexible, but inference for these models is challenging, particularly when the data features are complicated.

The latter is built on the asymmetric Laplace distribution (ALD) [9-11], and other parametric distributions, like an infinite mixture of Gaussian densities [12]. ALD, which is closely related to the check function for $\mathrm{QR}$, has been discussed in the literature $[6,9,11,13]$. A random variable $Y$ is said to follow ALD if its probability density function (pdf) with parameters $\mu, \sigma$ and $\tau$ is given by

$$
f(y \mid \mu, \sigma, \tau)=\frac{\tau(1-\tau)}{\sigma} \exp \left\{-\rho_{\tau}\left(\frac{y-\mu}{\sigma}\right)\right\},
$$

Where $\rho_{\tau}(u)=u(\tau-I(u<0))$ is the check function, $\mathrm{I}(\bullet)$ is the indicator function, $0<\tau<1$ is the skewness parameter, $\sigma>0$ is the scale parameter and $-\infty<\mu<\infty$ is the location parameter. The range of $\mathrm{y}$ is $(-\infty, \infty)$. We denote the above distribution by $\operatorname{ALD}(\mu, \sigma, \tau)$.

Briefly, if $\mathrm{Y} \sim \operatorname{ALD}(\mu, \sigma, \tau)$, then $P_{\mathrm{r}}(\mathrm{y} \leq \mu)=\tau$ and $P_{\mathrm{r}}(\mathrm{y}>\mu)=1-\tau$, which shows that the parameters $\mu$ and $\tau$ in ALD satisfy $\mu$ to be the $\tau$ th quantile of the distribution. However, the ALD is not smooth and thus difficult to maximize its likelihood function. Fortunately, as shown in these studies [14,15], the ALD has various mixture representations. A hierarchical mixture of exponential and normal distributions is utilized to develop algorithms for the QR models [14,15]. These important features of ALD have been generally adopted for likelihood based quantile inference, as well as the Bayesian inference. See $\mathrm{Yu}$ and Zhang's work [11] for further properties and generalizations of this distribution as well as its close relationship with QR. By utilizing this property, under independent data setting, a large number of QRbased statistical models and various associated analysis methods have been investigated in the literature. For example, a likelihood-based goodness-of-fit test has been proposed for QR [6]; Bayesian QR [9] , and the Bayesian estimation procedure for the Tobit QR model with censored data $[16,15]$, have also been developed.

Importantly, these two classes of QR inferential methods are not mutually exclusive. The relationship between the check function and ALD can be used to reformulate the QR method in the likelihood framework. Considering $\sigma$ a nuisance parameter, it can be easily shown that the minimization of equation (2) in the former method with respect to the parameter $\beta$ is exactly equivalent to the maximization of an ALD-based likelihood function in the latter.

It has been demonstrated that QR is widely used to analyze independent data in many important application areas. First, due to the importance of modeling extreme values accurately, the foreign direct investment (FDI), finance and economics are the most important area where QR is utilized. Girma and Gorg [1] and Zhou [2] used QR modeling to explore relationship between the foreign direct investment and economic growth. Several economists have examined wage structure and wealth distribution using QR [17-19]. Specifically, research has been conducted to explore the gap in wage and wealth distribution [20], including the effect of gender on wage $[21,22]$, wage differences between public and private entities [23-25], wealth inequality between urban and rural areas [26], and the impact of education on wage [27-29] and intergenerational earnings [30]. Focusing on the women group, Moshe Buchinsky studied female wage distribution in the USA [22] and their return to education [31] using QR as an analytic tool. In addition, QR has also been applied in economic-based discipline. In the area of economics and education, QR has been applied to examine the impact of school choice [32] and quality $[33,34]$ on student performance and achievement [33]. With the application of economics on management, QR has been used to study the effect of innovation on firm growth [35] and relationship between companys foreign ownership and production efficiency [36] as well as association of FDI and economic growth $[1,2]$. In the subarea of economics and policy, existing corruption levels have been explored [37] and the relationship between FDI and corruption level [38] has been examined using QR models. In the finance field, QR has been adopted to study housing price [39], capital structure [40], FDI and investment treaties [1] and stock market returns [41] based on cross-sectional data.

In addition, $\mathrm{QR}$ is becoming more popular in clinical, biomedical, and other health related research. For example, Austin et al. [42] examined the varying gender differences in the delivery of thrombolysis in patients with an acute myocardial infarction by QR. Briollais and Durrieu [43] provided a review of recent applications of QR to the area of genetics. Azagba and Sharaf [44] identified that increasing the intake of fruits and vegetables may be an effective dietary strategy to control weight and mitigate the risk of obesity, which is more effective at the higher quantiles of the body mass index (BMI) distribution. More applications of QR to independent data could be found in various fields, such as public health [45,46], bioinformatics [47], healthcare $[48,49]$, environmental science [50], and ecology [51,52].

\section{QR Models for Time-to-Event Data}

Time-to-event data arise when interest is focused on the time elapsing before an event is experienced. Application to analysis of this kind of data, called survival analysis or duration models, is objective to investigate the effects of covariates on the survival/duration time. These effects can be heterogeneous on low, medium, and high risk subjects. In other words, covariates may have greater effects at an early period of survival, and weaker effects or even no effect later, or vice versa. QR has been considered to apply to measure the differences of covariates effects at different quantiles of survival/duration time [53]. Furthermore, the survival/duration time often exists non-normality and long tails, and thus QR-based survival models provide more robust estimation than traditional mean regression-based ones.

Although, Coxs proportional hazard model is the most often used for survival analysis, it is rarely generalized to QR-based models. Alternatively, the accelerated failure time (AFT) model with the transformed survival time can be employed to QR field, in which logarithm transformation is the most commonly used one [53-56]. Due to the complexity of the time-to-event data, large number of studies has contributed to the QR-based AFT model under different scenarios. Ying et al. [57] studied a semiparametric procedure for median regression. Yang [54] extended the median regression with weighted empirical survival and hazard functions based estimation. Portnoy [58] generalized the principle of the Kaplan-Meier estimate under QR framework. Yin et al., [59] investigated the quantile regression model for correlated failure time data. Peng and Huang [60] developed an estimator which is very close to Nelson-Aalen estimator. Most recently, great work is still expanding this area to recurrent events [61-63], various censoring types [64-66], competing risks [65,67-69].

There are many applications of QR to survival analysis or duration models. For instance, in finance and economics, Schaech [70] assessed the association among bank liability structure and time to failure by a 
QR approach. In clinical research, Carey et al. [71] found that AIDS patients with lower growth velocity (below the 10th quantile) had significantly increased risk of death. In healthcare, Austin et al. [42] determined patient and system characteristics associated with the waiting time of essential medical treatment by QR, and found that gender had a greater impact upon those patients who had the greatest delays in treatment. Other interesting applications could also be found in economics [72-74], clinical and biomedical research [75,76], and healthcare areas $[77,78]$.

\section{QR Models for Longitudinal Data}

Longitudinal data, sometimes called panel data, show great complexity in statistical analysis and application due to the correlation between and within repeatedly measured observations. In statistics, mixed-effects models are becoming increasingly popular in longitudinal data analysis. However, the majority of longitudinal modeling methods are based on mean regression to concentrate only on the average effect of covariate and the mean trajectory of longitudinal outcome. Thus, mimic to independent data, QR has also been extended and applied to longitudinal data. Longitudinal QR has the capability, at both of the population and individual level, to identify heterogeneous covariates effects, and describe differences in longitudinal changes at different quantiles of the outcome, and provides more robust estimates when heavy tails and outliers exist.

Similar as QR for independent data, longitudinal QR models, specifically QR-based mixed-effects models have been proposed via different statistical approaches, which could also be classified into two categories: distribution-free and likelihood-based. In details, for example, Jung [79] firstly developed a quasi-likelihood method for median regression considering correlations between repeated measures for dependent data. He et al. [80] proposed a median regression based linear mixed-effects model for longitudinal data. Koenker [81] generalized his previous work on QR to longitudinal data via penalized least squares method. Other methods or algorithms used to QR includes Barrodale-Roberts algorithm [82], Expectation-Maximization (EM) algorithm [83], Monte Carlo Expectation-Maximization (MCEM) algorithm $[13,84,85]$, and Bayesian approach by Markov chain Monte Carlo (MCMC) procedure [86-93]. Longitudinal QR has been rapidly expanded in many areas, including investment and finance [94,95], economics [96], environmental science [97,98], geography [99], public health [100,101] and biomedical research [102-105]. In investment and finance areas, Bassett and Chen [94] utilized longitudinal QR to provide additional information from the time series data of portfolio returns based on the way style that affects returns at places other than the expected value of return. In economics, Buchinsky [96] studied US wage structure from 1963 to 1987 with the application of longitudinal QR. It provided a full scan of information among time effects, education level, and years of experience in different wage quantile. In public health, Smith et al. [100] revealed that the association between high blood pressure and living in an urban area has evolved from positive to negative, with the strongest changes occurring in the upper tail. In meteorology, Timofeev and Sterin [97] utilized longitudinal QR to analyze various changes in climate characteristics. In biomedical studies, Revzin et al. [104] investigated the effect of a naturally derived biological peptide, P28, and found that it produced slower rates of growth in the upper quantiles of melanoma tumor volumes in mice.

Data collected in many longitudinal studies record much information, not only repeated measures, but also time-to-event information. For example, in HIV/AIDS studies, viral load (the number of copies of HIV-1 RNA) and CD4 cell counts are important biomarkers of the severity of a viral infection, disease progression, and treatment evaluation, and their time trends of longitudinal measures may also be predictive of the risk of a terminal event. Thus, joint models are an active area of statistics, because of its capability on the bias reduction and improvement of estimates' efficiency. More recently, QR has been extended to more complicated joint models in AIDS research. Farcomeni and Viviani [85] developed QR-based longitudinalsurvival joint models in the presence of informative dropout. Huang et al. proposed QR-based mixed-effects joint models by considering many longitudinal data features simultaneously, including covariate measurement errors $[90,93,89]$, missing $[90,91]$, non-normality [9092], left-censoring [89,92], and time-to-event outcomes [91].

\section{Summary and Conclusion}

This review provided a general overview of QR-based models and methods targeting different types of data and application areas. We have illustrated that QR is a powerful tool to detect heterogeneous effects of covariates at different quantiles of the outcome, and complements excellently the mean regression when data are in presence of outliers and long tails. Recent developments and extensions in QR-based models offer increasing ability and flexibility in capturing independent, time-to-event, and longitudinal data with different data features, which can benefit applications in various scientific and finance areas.

We believe that $\mathrm{QR}$, a comprehensive strategy, has a bright future. In financial/investment market, $\mathrm{QR}$ is more powerful for investors to predict investment strategies; in medicine, according to the idea of "precision medicine", QR is more precise for physicians to evaluate treatment and make clinical decisions, compared to mean regression models. In statistics, especially in the "big data" era, data sources get richer, data structures become more complicated, extreme values and heterogeneity increase. Instead of the mean regression, which hardly meets our expectation, QR methods dig deeper into the data, grab more information, and become more relevant. Last but not the least, as the power of the computer has advanced, the computational load for QRbased models and methods has decreased substantially. Thus, more complicated QR-based models could be considered under a Bayesian framework [89-93] and applied to more diverse areas in near future.

A final note that we would like to make is possible software to implement QR modeling methods. The most widely used software for QR models is R with "quantreg” package [106]. It covers linear, nonlinear parametric and non- parametric (total variation penalized) models for conditional quantiles of a univariate response, and several methods for handling censored time-to-event data. Other $\mathrm{R}$ packages are also available for specific $\mathrm{QR}$ topics. For example, $\mathrm{R}$ package "cmprskQR" [69] is developed for analysis of competing risks using QR; package "lqmm" [107], and "qrLMM" [108], deal mainly with longitudinal data via QR-based linear or non-linear mixed-effects models. SAS currently also includes a "quantreg" procedure, which is similar as the R "quantreg" package. Stata software has "qreg” function to fit QR models, but the capabilities are limited. QR also has been added to SPSS (version 22.0.0 or later), just simply estimate one or more conditional quantiles for a linear model. When the model components are very complicated, especially for survival and longitudinal data with multiple data features, which bring extremely heavy computational load, the Bayesian method shows its advantages. The WinBUGS software [109] interacted with the package "R2WinBUGS" in R and "Rstan" package [110] in R are good choices with a lot of flexibility for Bayesian inference. 
Citation: Huang Q, Zhang H, Chen J, He M (2017) Quantile Regression Models and Their Applications: A Review. J Biom Biostat 8: 354. doi: 10.4172/2155-6180.1000354

\section{Acknowledgements}

This research is partially supported by the National Natural Science Foundation of China (81671633) to J. Chen.

\section{References}

1. Girma S, Gorg H (2002) Foreign direct investment spillovers and absorptive capacity evidence from quantile regressions. Leverhulme Centre for Research on Globalisation and Economic Policy(14).

2. Zhou C (2011) A quantile regression analysis on the relations between foreign direct investment and technological innovation in china. International Conference of Information Technology Computer Engineering and Management Sciences 4: 28-41.

3. Collins FS, Varmus $H$ (2015) A new initiative on precision medicine. New England Journal of Medicine 372: 793-795.

4. Mirnezami R, Nicholson J, Darzi A (2012) A Preparing for precision medicine New England Journal of Medicine 366: 489-491.

5. Koenker R, Bassett JRG (1978) Regression quantiles Econometrical. Journal of the Econometric Society 46: 33-50.

6. Koenker R, Machado JA (1999) Goodness of fit and related inference processes for quantile regression. Journal of the American Statistical Association 94 1296-1310.

7. Koenker R, Hallock K (2001) Quantile regression an introduction. Journal of Economic Perspectives 15: 43-56.

8. Koenker R (2005) Quantile regression Cambridge University Press 38.

9. Yu K, Moyeed RA Bayesian (2001) quintile regression. Statistics \& Probability Letters 54: 437-447.

10. Yu K, Lu Z, Stander J (2003) Quantile regression applications and curren research areas. Journal of the Royal Statistical Society Series D (The Statistician) 52: 331-350

11. Yu K, Zhang J (2005) A three-parameter asymmetric Laplace distribution and its extension. Communications in Statistics Theory and Methods 34: 18671879.

12. Reich BJ, Bondell HD, Wang HJ (2010) Flexible bayesian quantile regression for independent and clustered data. Biostatistics 2010 11: 337-352.

13. Geraci M, Bottai M (2007) Quantile regression for longitudinal data using the asymmetric laplace distribution. Biostatistics 8: 140-154.

14. Kotz S, Kozubowski TJ, Podg orski K (2001) Asymmetric multivariate laplace distribution. The Laplace Distribution and Generalizations 239-272.

15. Kozumi H, Kobayashi G (2011) Gibbs sampling methods for Bayesian quintile regression. Journal of Statistical Computation and Simulation 81:1565-1578.

16. Yu K, Stander J (2007) Bayesian analysis of a Tobit quantile regression model. Journal of Econometrics 137: 260-276.

17. Machado JA, Mata J (2005) Counterfactual decomposition of changes in wage distributions using quintiles regression. Journal of Applied Econometrics 20: $445-465$

18. Buchinsky M (1995) Quantile regression box-cox transformation model and the us wage structure 1963-1987. Journal of Econometrics 65: 109-154

19. Chernozhukov V, Hansen (2004) The effects of 401 (k) participation on the wealth distribution an instrumental quantile regression analysis. Review of Economics and Statistics 86: 735-751.

20. Melly B (2005) Decomposition of differences in distribution using quintiles regression. Labor Economics 12: 577-590.

21. Garcia J, Hernandez PJ, Lopez-Nicolas (2002) A How wide is the gap? an investigation of gender wage differences using quantile regression. Economic Applications of Quantile Regression. Springer 149-167.

22. Buchinsky M (1998) The dynamics of changes in the female wage distribution in the USA a quantile regression approach. Journal of Applied Econometrics $1-30$.

23. Melly B (2005) Public-private sector wage differentials in Germany Evidence from quantile regression. Empirical Economics 30: 505-520.

24. Poterba JM, Rueben KS (1994) The distribution of public sector wage premia new evidence using quantile regression methods. Technical Report National Bureau of Economic Research.

25. Nielsen HS, Rosholm M (2002) The public-private sector wage gap in Zambia in the 1990 A quantile regression approach. Economic Applications of Quantile Regression Springer 169-182.

26. Nguyen BT, Albrecht JW, Vroman SB, Westbrook MD (2007) A quantile regression decomposition of urban-rural inequality in Vietnam. Journal of Development Economics 2007 83: 466-490.

27. Martins PS, Pereira PT (2007) Does education reduce wage inequality? quantile regression evidence from 16 countries. Labour Economics 11: 355-371.

28. Hartog J, Pereira PT, Vieira JA (2001) Changing returns to education in Portugal during the 1980s and early 1990s Old and quantile regression estimators. Applied Economics 33: 1021-1037.

29. Guinness S, Bennett J (2007) Over education in the graduate labour market $A$ quantile regression approach. Economics of Education Review 26: 521-531.

30. Eide ER, Showalter MH (1999) Factors affecting the transmission of earnings across generations a quintile regression approach. Journal of Human Resources 253-267.

31. Buchinsky M (2002) Quantile regression with sample selection Estimating women return to education in the US. Economic Applications of Quantile Regression Springer 87-113.

32. Harding M, Lamarche C (2009) A quantile regression approach for estimating panel data models using instrumental variables. Economics Letters 104: 133-135.

33. Eide E, Showalter MH (1998) The effect of school quality on student performance. A quantile regression approach Economics Letters 58: 345-350.

34. Levin J (2002) For whom the reductions count A quantile regression analysis of class size and peer effects on scholastic achievement. Economic Applications of Quantile Regression Springer 221-246.

35. Coad A, Rao R (2008) Innovation and firm growth in high-tech sectors. A quantile regression approach Research Policy 37: 633-648.

36. Dimelis S, Louri H (2002) Foreign ownership and production efficiency a quantile regression analysis. Oxford Economic Papers 54: 449-469.

37. Billger SM, Goel RK (2009) Do existing corruption levels matter in controlling corruption? Cross-country quantile regression estimates. Journal of Development Economics 90: 299-305.

38. Okada K, Samreth S (2012) The effect of foreign aid on corruption A quantile regression approach. Economics Letters 115: 240-243.

39. Zietz J, Zietz E, Sirmans GS (2008) Determinants of house prices a quantile regression approach. The Journal of Real Estate Finance and Economics 37: 317-333.

40. Fattouh B, Scaramozzino P, Harris L (2005) Capital structure in south korea a quantile regression approach. Journal of Development Economics 76: 231-250.

41. Barnes ML, Hughes ATW (2002) A quantile regression analysis of the cross section of stock market returns.

42. Austin PC, Tu JV, Daly PA, Alter DA (2005) The use of quantile regression in health care research a case study examining gender differences in the timeliness of thrombolytic therapy. Statistics in Medicine 24: 791-816.

43. Briollais L, Durrieu G (2014) Application of quantile regression to recent genetic and omic studies. Human Genetics 133: 951-966.

44. Azagba S, Sharaf MF (2012) Fruit and vegetable consumption and body mass index a quantile regression approach. Journal of Primary Care \& Community Health 3: $210-220$.

45. Ellerbe CN, Gebregziabher M, Korte JE, Mauldin J, Hunt KJ (2013) Quantifying the impact of gestational diabetes mellitus maternal weight and race on birth weight via quantile regression. PLoS ONE 8: 65-117.

46. Peterson MD, Krishnan C (2015) Growth charts for muscular strength capacity with quantile regression. American Journal of Preventive Medicine 49: 935-938.

47. Song X, Li G, Zhou Z, Wang X, lonita-Laza I, et al.( 2017) A novel quantile regression tool for extol discovery. Bioinformatics.

48. Sherwood B, Wang L, Zhou XH (2013) Weighted quantile regression for analyzing health care cost data with missing covariates. Statistics in Medicine 32: 4967-4979. 
Citation: Huang Q, Zhang H, Chen J, He M (2017) Quantile Regression Models and Their Applications: A Review. J Biom Biostat 8: 354. doi: 10.4172/2155-6180.1000354

Page 5 of 6

49. Cook BL, Manning WG (2009) Measuring racial/ethnic disparities across the distribution of health care expenditures. Health Services Research 44: 1603-1621.

50. Borgoni R (2011) A quantile regression approach to evaluate factors influencing residential indoor radon concentration. Environmental Modeling \& Assessment 16: $239-250$

51. Knight CA, Ackerly DD (2002) Variation in nuclear DNA content across environmental gradients a quantile regression analysis. Ecology Letters 5: 66-76.

52. Cade BS, Noon BR (2003) A gentle introduction to quantile regression for ecologists. Frontiers in Ecology and the Environment 1: 412-420.

53. Koenker R, Geling O (2001) Reappraising medfly longevity a quantile regression survival analysis. Journal of the American Statistical Association 96: 458-468.

54. Yang S (1999) Censored median regression using weighted empirical survival and hazard functions. Journal of the American Statistical Association 94: 137-145.

55. Kottas A, Gelfand AE (2001) Bayesian semi parametric median regression modeling. Journal of the American Statistical Association 96: 1458-1468.

56. Fitzenberger B, Wilke RA (2006) Using quantile regression for duration analysis. Modern Econometric Analysis Springer 103-118.

57. Ying Z, Jung SH, Wei LJ (1995) Survival analysis with median regression models. Journal of the American Statistical Association 90: 178-184.

58. Portnoy S (2003) Censored regression quantiles. Journal of the American Statistical Association 98: 1001-1012.

59. Yin G, Cai J (2005) Quantile regression models with multivariate failure time data. Biometrics 61: 151-161.

60. Peng L, Huang $Y(2008)$ Survival analysis with quantile regression models. Journal of the American Statistical Association 103: 637-649.

61. Sun X, Peng L, Huang Y, Lai HJ (2016) Generalizing quantile regression for counting processes with applications to recurrent events. Journal of the American Statistical Association 111: 145-156.

62. Sun L, Zhou X, Guo S (2011) Marginal regression models with time varying coefficients for recurrent event data. Statistics in Medicine 30: 2265- 2277

63. Luo X, Huang CY, Wang L (2013) Quantile regression for recurrent gap time data. Biometrics 69: 375-385

64. Portnoy S, Lin G (2013) Asymptotic for censored regression quantiles. Journal of Nonparametric Statistics 22: 115-130.

65. Fan C, Zhang F, Zhou Y (2016) Power transformed linear regression on quantile residual life for censored competing risks data. Communications in Statistics-Theory and Methods 45: 5884-5905.

66. Xue X, Xie X, Strickler HD (2016) A censored quantile regression approach for the analysis of time to event data. Statistical Methods in Medical Research.

67. Peng L, Fine JP (2016) Competing risks quantile regression. Journal of the American Statistical Association104: 1440-1453.

68. Hsieh JJ, Wang HR (2016) Quantile regression based on counting process approach under semi-competing risks data. Annals of the Institute of Statistical Mathematics 1-25.

69. Dlugosz S, Lo SM, Wilke RA (2017) Competing risks quantile regression at work indepth exploration of the role of public child support for the duration of maternity leave. Journal of Applied Statistics 44: 109-122.

70. Schaeck K (2008) Bank liability structure flick loss and time to failure A quantile regression approach. Journal of Financial Services Research 33: 163-179.

71. Carey VJ,Yong F, Frenkel LM ,McKinney R (2004) Growth velocity assessment in paediatric aids smoothing penalized quantile regression and the definition of growth failure. Statistics in Medicine 23: 509-526.

72. Fitzenberger B, Koenker R, Machado JA (2013) Economic applications of quantile regression. Springer Science \& Business Media.

73. Koenker R, Bilias $Y$ (2002) Quantile regression for duration data a reappraisal of the Pennsylvania reemployment bonus experiments. Economic Applications of Quantile Regression Springer 199-220.

74. Koenker R, Park BJ (1996) An interior point algorithm for nonlinear quantile regression. Journal of Econometric 71: 265-283.

75. Flemming JA, Nanji S, Wei X, Webber C, Groome P, et al. (2017) Association between the time to surgery and survival among patients with colon cancer $A$ population based study. European Journal of Surgical Oncology.

76. Faradmal J, Roshanaei G, Mafi M, Sadighi-Pashaki A, Karami M (2016) Application of censored quantile regression to determine overall survival related factors in breast cancer. Journal of Research in Health Sciences 16: 36-40.

77. Ding R, McCarthy ML, Desmond JS, Lee JS, Aronsky D, et al. (2010) Characterizing waiting room time treatment time and boarding time in the emergency department using quantile regression. Academic Emergency Medicine 17: 813-823.

78. Cournane S, Conway R, Creagh D, Byrne D, Sheehy N, et al. (2016) Radiology imaging delays as independent predictors of length of hospital stay for emergency medical admissions. Clinical Radiology 71: 912-918.

79. Jung SH (1996) Quasi-likelihood for median regression models. Journal of the American Statistical Association 91: 251-257.

80. He X, Fu B, Fung WK (2003) Median regression for longitudinal data. Statistics in Medicine 22: 3655-3669.

81. Koenker R (2004) Quantile regression for longitudinal data. Journal of Multi variate Analysis $91: 74-89$

82. Wang HJ, Fygenson M (2009) Inference for censored quantile regression models in longitudinal studies. The Annals of Statistics 37: 756-781.

83. Farcomeni A (2012) Quantile regression for longitudinal data based on laten Markov subject-specific parameters. Statistics and Computing 22: 141-152.

84. Liu Y, Bottai M (2009) Mixed-effects models for conditional quantiles with longitudinal data. The International Journal of Biostatistics 5 .

85. Farcomeni A,Viviani S (2015) longitudinal quantile regression in the presence of informative dropout through longitudinal survival joint modeling. Statistics in Medicine 34: 1199-1213.

86. Yuan $Y$, Yin $\mathrm{G}$ (2010) Bayesian quantile regression for longitudinal studies with non ignorable missing data. Biometrics 66: 105-114.

87. Luo Y, Lian H, Tian M (2012) Bayesian quantile regression for longitudinal data models. Journal of Statistical Computation and Simulation 82: 1635-1649.

88. Kim MO, Yang Y (2012) Semi parametric approach to a random effects quantile regression model. Journal of the American Statistical Association 106 1405-1417.

89. Tian Y, Tian M (2015) Bayesian joint quantile regression for mixed effects models with censoring and errors in covariates. Computational Statistics 31: 1031-1057.

90. Huang Y (2016) Quantile regression-based Bayesian semi parametric mixed effects models for longitudinal data with non normal missing and measured covariate. Journal of Statistical Computation and Simulation 86: 1183-1202.

91. Huang Y, Chen J (2016) Bayesian quantile regression based nonlinear mixed effects joint models for time to event and longitudinal data with multiple features. Statistics in Medicine 35: 5666-5685.

92. Huang Y, Chen J, Lu X (2016) Bayesian approach to nonlinear mixed effects quantile regression models for longitudinal data with non-normality and left-censoring. Journal of Advanced Statistics 1: 109.

93. Huang Y, Chen J, Qiu H (2016) Bayesian quantile regression for nonlinear mixed effects joint models for longitudinal data in presence of measured covariate errors. Journal of Biopharmaceutical Statistics.

94. Bassett GW,Chen HL (2002) Portfolio style Return based attribution using quantile regression. Economic Applications of Quantile Regression 293-

95. Taylor JW (1999) A quantile regression approach to estimating the distribution of multi period returns. The Journal of Derivatives 7: 64-78.

96. Buchinsky M (1994) Changes in the us wage structure 1963-1987 Application of quantile regression. Econometrical journal of the Econometric Society $405-458$

97. Timofeev A, Sterin A (2010) Using the quantile regression method to analyze changes in climate characteristics. Russian Meteorology and Hydrology 35: 310-319.

98. Bind MA, Peters A, Koutrakis P, Coull B, Vokonas P, et al. (2016) Quantile regression analysis of the distributional effects of air pollution on blood 
Citation: Huang Q, Zhang H, Chen J, He M (2017) Quantile Regression Models and Their Applications: A Review. J Biom Biostat 8: 354. doi: 10.4172/2155-6180.1000354

Page 6 of 6

pressure heart rate variability blood lipids and biomarkers of inflammation in elderly american men the normative aging study. Environmental Health Perspectives 124: 1189.

99. Cromley RG, Hanink DM, Bentley GC (2012) A quantile regression approach to areal interpolation. Annals of the Association of American Geographers 102: 763-777.

100. Smith LB, Fuentes M, Gordon-Larsen P, Reich BJ (2015) Quantile regression for mixed models with an application to examine blood pressure trends in china. The Annals of Applied Statistics 9: 1226.

101. Bottai M, Frongillo EA, Sui X, O'Neill JR, McKeown RE, et al. (2014) Use of quantile regression to investigate the longitudinal association between physical activity and body mass index. Obesity 22 .

102. Lachos VH, Chen MH, Abanto-Valle CA, Azevedo CL (2015) Quantile regression for censored mixed-effects models with applications to HIV studies. Statistics and Its Interface 8: 203.

103. Li C, Dowling NM, Chappell R (2015) Quantile regression with a change point model for longitudinal data An application to the study of cognitive changes in preclinical Alzheimer's disease. Biometrics 71: 625-635.
104. Revzin E, Majumdar D, Bassett GW (2014) Conditional quantile regression models of melanoma tumor growth curves for assessing treatment effect in small sample studies. Statistics in Medicine 33: 5209-5220.

105.Patel DE, Geraci M, Cortina-Borja M (2016) Modeling normative kinetic perimeter isopters using mixed effects quantile regression. Journal of Vision 16: 7-17.

106. Koenker R (2013) Quantreg Quantile regression package version $5.05 \mathrm{R}$ Foundation for Statistical Computing Vienna.

107. Geraci M (2014) Linear quantile mixed models the lqmm package for Laplace quantile regression. Journal of Statistical Software 57: 1-29.

108. Galarza CE, Galarza MCE (2015) Package qrlmm. Communications in Statistics-Theory and Methods 34: 1867-1879.

109.Lunn DJ, Thomas A, Best N, Spiegelhalter D (2000) Winbugs a bayesian modelling framework concepts structure and extensibility. Statistics and Computing 10: 325-337.

110. Carpenter B, Gelman A, Hoffman MD, Lee D, Goodrich B, et al. (2017) A probabilistic programming language. Journal of Statistical Software 76: 1-32. 